\title{
HIGH-RESOLUTION NUMERICAL METHODS FOR COMPRESSIBLE MULTI-PHASE FLOW IN HIERARCHICAL POROUS MEDIA
}

Progress Report

September 1993 - September 1994

John A. Trangenstein

Department of Mathematics

Duke University

Box 90320

Durham, NC 27708-0320

March 15, 1994

FECENGO

Mik $1 \times 1994$

Os r

\section{PREPARED FOR THE NATIONAL SCIENCE FOUNDATION \\ UNDER GRANT NUMBER SES-DMS-9201361 \\ AND FOR THE DEPARTMENT OF ENERGY \\ UNDER GRANT DE-FG05-92ER25145, A001}

\section{DISCLAIMER}

This report was prepared as an account of work sponsored by an agency of the United States Government. Neither the United States Government nor any agency thereof, nor any of their employees, makes any warranty, express or implied, or assumes any legal liability or responsibility for the accuracy, completeness, or usefulness of any information, apparatus, product, or process disclosed, or represents that its use would not infringe privately owned rights. Reference herein to any specific commercial product, process, or service by trade name, trader.ark, manufacturer, or otherwise does not necessarily constitute or imply its endorsement, recommendation, or favoring by the United States Government or any agency thereof. The views and opinions of authors expressed herein do not necessarily state or reflect those of the United States Government or any agency thereof. 


\section{Personnel}

This research program is being conducted by several people:

John Trangenstein is the principal investigator, and collaborates on all of the projects. During FY94, he has been supported by the Defense Nuclear Agency for work in adaptive mesh refinement for wave propagation in elastic-plastic solids, and by NSF grant DMS-9201034 (from AFOSR funds) for work with David Schaeffer and others on front tracking and adaptive mesh refinement for shear bands in granular solids. John Trangenstein received no salary support from the grants covered by this report during FY 93 or 94.

Sameer Khan is a post-doctoral fellow in Mathematics at Duke. He received his Ph.D. in Petroleum Engineering at the University of Texas in December 1992 , and is supported jointly by these two grants.

John Davies is the systems programmer in the Mathematics Department at Duke. He is supported jointly by these two grants, by NIH grants awarded to Michael Reed and Harold Layton, and by the mathematics department.

Richard Hornung is a doctoral student in mathematics at Duke. He is supported by these two grants.

Khaled Furati is a doctoral student in mathematics at Duke. He is supported by a fellowship from Saudi Arabia.

Jon Simons is an undergraduate in Computer Science at Duke.

\section{Technical Progress}

This is the second year in the proposed three-year effort to develop high-resolution numerical methods for multi-phase flow in hierarchical porous media. The issues being addressed in this research are:

Computational efficiency: Field-scale simulation of enhanced oil recovery, whether for energy production or aquifer remediation, is typically highly under-resolved. This is because rock transport properties vary on many scales, and because current numerical methods have low resolution.

Effective media properties: Since porous media are formed through complex geologic processes, they involve significant uncertainty and scaledependence. Given this uncertainty, knowledge of ensemble averages of flow in porous media can be preferable to knowledge of flow in specific realizations of the reservoir. However, current models of effective properties do not represent the observed behavior very well. Relative permeability models present a good example of this problem. In practice, these models 
seldom provide realistic representations of hysteresis, interfacial tension effects or three-phase flow; there are no models that represent well all three effects simultaneously.

Wave propagation: It is common in the petroleum industry to assume that the models have the same well-posedness properties as the physical system. An example of this fallacy is given by the three-phase relative permeability models; they were widely assumed by the petroleum community to produce hyperbolic systems for the Buckley-Leverett equations, but later the mathematics community proved that these models inherently produce local elliptic regions. Since numerical methods must use the models for computations, oscillations could erroneously be attributed to numerical error rather than modeling difficulties. When wave propagation is well-understood by analysis, it can provide useful information about the expected behavior of the flows, and can assist in the development of high-resolution numerical methods.

During this year, we have concerned ourselves with the following tasks aimed at addressing the previous issues:

Surfactant model development: This model was chosen because of its applicability to both contaminant cleanup and enhanced oil recovery, its rich representation of physical processes, and the challenge of applying highresolution numerical methods to it. In our formulation, this model involves seven components and from one to three phases. This makes it one of the most complicated models to which modern shock-capturing methods and adaptive mesh refinement have been applied.

Elliptic adaptive mesh refinement: This task is designed to extend adaptive mesh refinement in a new and important direction. Since multidimensional flow problems involve both hyperbolic behavior (through the transport of chemical species) and elliptic / parabolic behavior (through the pressure gradient involved in Darcy transport), it is reasonable to develop numerical methods that are well-suited to each type of behavior. Efficient treatment of hyperbolic conservation laws has been achieved through a combination of high-resolution shock-capturing schemes and adaptive mesh refinement. We are examining methods to extend the benefits of adaptive mesh refinement to the elliptic / paraboilc aspects of multi-dimensional flow in porous media. The approach we have chosen to handle this adaptive mesh refinement is particularly well-suited to a numerical study of effective media properties.

Analysis of wave behavior: Our third task addresses some analytical questions about a simple surfactant model, and hysteresis in relative permeabilities. solutions. Recent mathematical analysis by Johansen has raised the possibility that initial-value problems for the surfactant model have multiple solutions. The resolution of this problem can be obtained by 
understanding the evolution of waves in this model. Enhanced oil recovery models, such as the polymer and surfactant models, lead to the development of oil banks in enhanced oil recovery processes subject to continuous injections (Riemann problems). These oil banks correspond to non-monotone behavior in the saturations, and therefore trigger hysteresis. We have developed analytical solutions to these problems, both to understand the effect on the physical predictions, and to determine useful test pioblems for our numerical methods.

3D computation: Our fourth task involves the development of second-order Godunov methods for three-dimensional polymer flooding. Because of the intense computational requirements of three-dimensional simulation, the use of higl-resolution numerical methods is particularly important to computational efficiency.

Visualization: We have developed effective visualization techniques for our simulators. These methods are useful for understanding fluid flow behavior, debugging, and collaboration at a distance.

We will now describe each of these tasks in detail.

\subsection{Surfactant Model}

The surfactant model we are studying was developed over the past decade by Gary Pope and his students at the University of Texas Department of Petroleum Engineering. It models the micellar-polymer flooding process, which typically involves at least five stages:

Preflush: Since salinity can significantly lower the effectiveness of surfactants, if necessary the reservoir is first flushed with a fluid, such as water, to lower the salinity

Slug: The injection of surfactant lowers the interfacial tension between oil and water, leading to a nearly complete sweep of oil from the pore spaces. This in turn leads to the development of an oil bank ahead of the surfactant. Often alcohol is injected with the surfactant to adjust the phase behavior.

Mobility buffer: In order to drive the slug across the reservoir, a fluid with viscosity comparable to that of the hydrocarbon is used. Typically, this fluid is a mixture of water and polymer. This serves to prevent the fluids in the remaining stages from developing viscous instabilities that channel through the slug and destroy the efficiency of the recovery process.

Taper: In this stage, the polymer in the mobility buffer is gradually reduced. The engineering principle here is based on numerical simulation with loworder methods, which showed the possibility of recovery failure due to viscous instabilities that develop at the trailing edge of narrowly-tapered mobility buffers and punch through the slug. There is evidence from higher- 
resolution simulations that these tapers do not need to be as large as first thought.

Chase water: This is an inexpensive injection fluid, such as water.

This process is being used for enhanced oil recovery by Statoil, and has been examined by a number of the domestic oil companies in order to continue production from the mature domestic reservoirs. This process is also under study by Linda Abriola, of the Civil Engineering Department at the University of Michigan, for the removal of hydrocarbon contaminants from aquifers. Drs. Abriola and Pope are currently funded jointly by the EPA to study the potential success of this process.

The surfactant model has led to the development of the UTCHEM simulator, which is distributed to essentially all of the domestic oil companies, as well as a number of foreign companies, through the Center for Petroleum and Geosystems Engineering (CPGE) at UT. This simulator is based on a fixed mesh, finite difference scheme using higher-order differencing in at most one or two variables (the relative permeabilities) along coordinate directions. The scheme is first-order in multiple dimensions, and whenever there are waves in addition to the Buckley-Leverett waves. It also requires timesteps that are significantly smaller than the Courant-Friedrich-Levy condition for numerical stability. This simulator is being modified for parallelization under a DOE HPCC grant jointly awarded to Gary Pope, and Mary Wheeler at the Department of Mathematical Sciences at Rice University.

We chose to study this model because it is one of the few enhanced oil recovery techniques that are being considered for use in aquifer remediation. (Another is steam flooding.) Thus we have the potential to influence two DOE missions at the same time, namely environment cleanup and energy production.

In FY 93 we completely reprogrammed the phase behavior model in UTCHEM. The purpose of the model revision was to remove some obvious discontinuities and to improve the numerical performance. The revised model was made available to Gary Pope, and may be transmitted on to the industrial subscribers of CPGE. We also developed a numerical strategy for dealing with the mass conservation equations, which are subject to the constraint that the sum of the volume-occupying component concentrations is one. This led to the development of a one-dimensional simulator, based on a second-order Godunov method.

In FY 94 we aicomplished the following:

Simulations We ran a number of simulations in $1 D$ and 2D with the secondorder Godunov surfactant flooding simulator.

- The fluid was assumed to be incompressible, so in 1D we used a constant total fluid velocity and pressure, avoiding the need for the solution of an elliptic pressure equation. As a result, we were able to use our existing adaptive mesh refinement library in combination with the surfactant flooding model. The $2 \mathrm{D}$ simulations were run 
with a fixed mesh. Some example simulation results are shown in the accompanying figures.

- Figure 1 shows total concentrations of various components (vater, oil, surfactant, alcohol, polymer, anions and cations) in a one-dimentional simulation using adaptive mesh-refinement. Notice the formation of the oil bank (shown in blue) which is being followed by surfactant (green) and polymer slugs (cyan, which prints as a light blue). The left side of the surfactant slug involves transitions between a number of wave families, and is very difficult to resolve with first-order methods.

- Figure 2 shows color contours of satvrations, pressure, phase index, effective salinity and total concentrations for a similar process in two dimensions on a 40 by 20 mesh. Here it is more difficult to visualize the complicated chemistry during the flow. We show color profiles, using a hot-cold color map (red = high, blue = low), of the six components ("totconc" = water, "totconc2" = oil, "totconc3" = surfactant, "totconc5" = polymer, "totconc6" = anions, "totconc7" = cations), the three saturations ("sat" = aqueous, "sat2" = oleic, "sat3" = microemulsion), the pressure, and the number of phases (oleic-microemulsion in blue, three-phases in red in the middle, aqueous-microemulsion in green, and aqueous-oleic in red at the upper right corner). The effect of gravity is quite prominent here. For example, the contours of oil concentrations (totconc2) show high oil concentrations (shown in red) at top of the reservoir due to the lower oil density.

Model developments Other simulations indicated that some more subtle discontinuities were still present in the model. For example, while the relative permeability curves for aqueous and oleic phases are typically convex, the relative permeability curves for microemulsion phases are typically concave. At the residual microemulsion saturation, the slope of the relative permeability is large; in the version of the model used in FY 93 it would be infinite. Another difficulty occured when plait points were located at the corners of the two-phase regions; in this case the compositional derivative of the interfacial tension was undefined. These problems were due to the specific implementation of the previous model; other choices of the input parameters would have led to reasonable predictions. As a result, in FY 94 we undertook another complete rewrite of the phase behavior model. The current version of the model is described in the accompanying draft of a joint paper with Gary Pope and Sameer Khan. In addition to removing the remaining discontinuities in the previous model, we also took care to treat general permeability tensors in a way that is invariant under coordinate transformations. We also added rate-dependent dissolution to the model. 


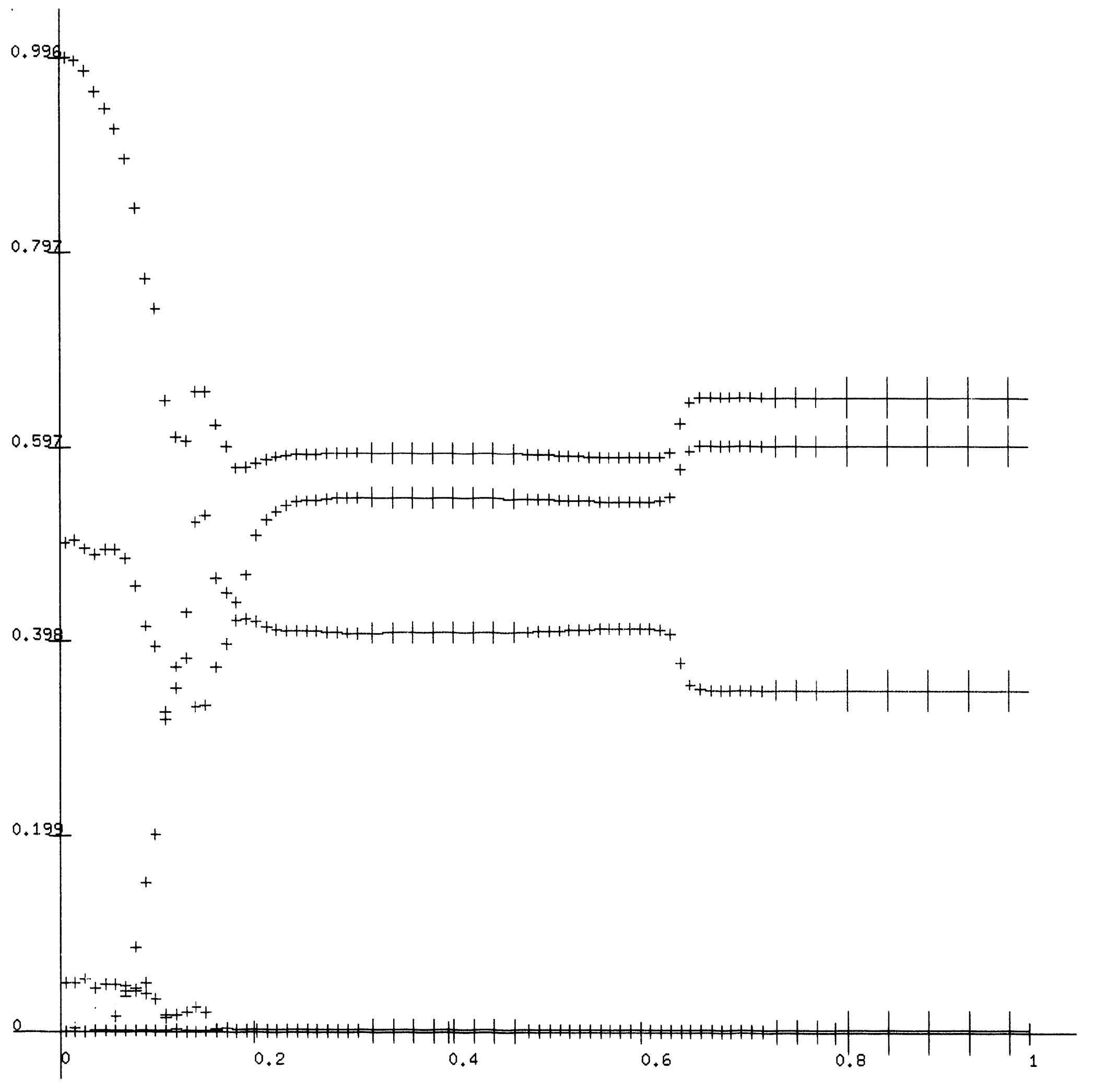




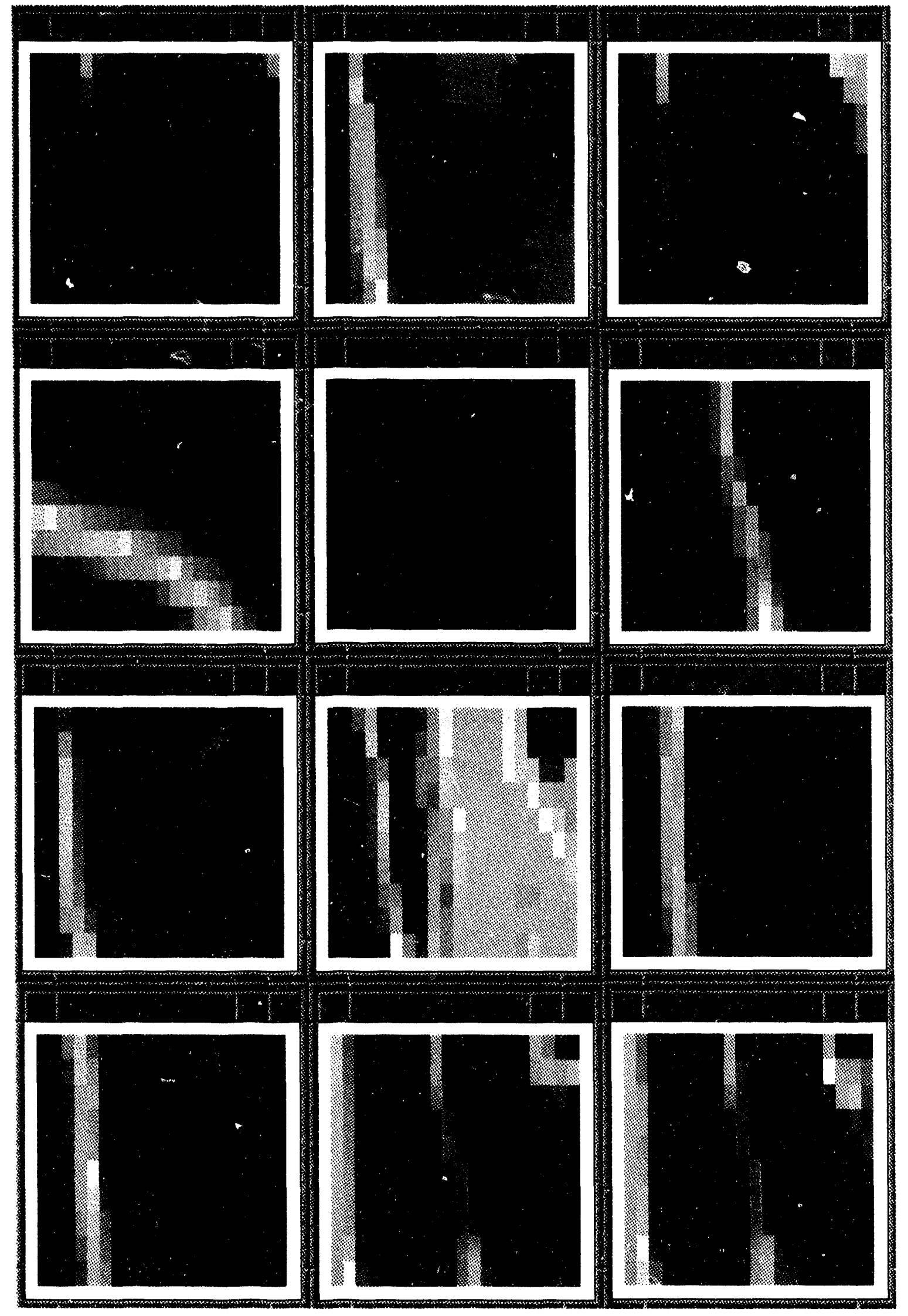


Adaptive mesh refinement In the remainder of FY 94 we will be testing the revised model and combining it with the multi-level multigrid algorithm for the pressure equation. (See the section on Elliptic Adaptive Mesh Refinement below.) We will then be able to run 2D simulations using adaptive mesh refinement.

Analysis . In FY 94 wa also made some additional progress in understanding the analytical structure of the characteristic speeds for this surfactant model. One of the characteristic speeds is associated with the ion exchange model, and is a weighted average of the fluxes of water and surfactant. Another characteristic speed is associated with the concentration of the anions, and is a ratio of the component flux of water to the component flux of surfactant. The reinaining characteristic speeds depend on the number of phases being formed. For single phase flow, two of the characteristic speeds are the particle velocity. The other characteristic speeds are the particle velocity times retardation factors, due to the rates of change of polymer adsorption and surfactant adsorption. For three-phase flow, two of the characteristic speeds are the familiar Buckley-Leverett speeds, which are eigenvalues of the matrix of partial derivatives of the phase velocities with respect to the phase saturations. The remaining two characteristic speeds are weighted averages of particle velocities, with weights related to the the rate of change of the phase concentrations with respect to the alcohol partitioning coefficients, and related to the rate of change of the flux of water with respect to the polymer concentration. The two-phase characteristic speeds are the most difficult to determine, especially since there are so many different two-phase mixtures in the model The analysis of the two-phase cases will be continued later in FY 94, once the 2D simulation techniques are fully developed.

The work on the surfactant model is being conducted jointly by John Trangenstein and Sameer Khan.

\subsection{Elliptic Adaptive Mesh Refinement}

Flow in porous media involves both hyperbolic and elliptic/parabolic behavior. While fluid fronts move slowly (on the order of a foot per day), pressure effects move rapidly (on the order of a mile per day). The pressure effects are typically modeled by summing the concentration conservation equations to obtain a divergence-free condition on the total fluid velocity, and then applying Darcy's law to obtain an elliptic pressure equation. Compressible flow is slightly more complicated, and will be treated in a later stage of this project.

While adaptive mesh refinement is reasonably well-understood for the conservation law, the use of adaptively refined meshes for equations with mixed character is more problematical. In order to provide a pressure and total fluid velocity to the conservation law, it is necessary to solve an elliptic pressure equation on the adaptively refined mesh. There are some special considerations here. 
First of all, the discrete gradient in the pressure equation needs to be the same as the gradient used in the conservative difference. This is necessary so that the discretization of the conservation law is consistent with the discretization of the pressure equation; in particular it is important that the sum of the component fluxes in the conservation law is equal to the total flow rate used in the pressure equation. Secondly, the iterative techniques should be as efficient as possible, in order to prevent the pressure equation from totally dominating the computational effort. Thirdly, it would be helpful if the iterative technique were designed to assist in the numerical determination of effective media parameters.

Our approach is to use the same adaptive meshes for the pressure equation and the conservation law. This is reasonable, so that the pressure equation can provide accurate velocities to the conservation law, and so that the conservation law can provide accurate transport properties to the pressure equation. We are using adaptively refined meshes consisting of hierarchical overlying rectangular grid patches. Thus we solve the pressure equation on a composite mesh, involving both coarse and fine grid cells.

Note that our use of hierarchical, overlying meshes is akin to the view of porous media as involving a hierarchy of scales. The effective properties of the porous medium, such as permeability and porosity, depend on the scale at which they are viewed. Various approaches by engineers and physicists have been developed to determine these effective media properties. We will be testing these ideas as potential averaging operators in our multi-grid iteration.

We use domain decomposition iterative techniques on patches at the same level of refinement, and to use multi-grid iterative techniques to communicate information between different levels of refinement. Domain decomposition is naturally suited to our use of rectangular arrays of grid cells, and allows the use of fast iterative methods on structured grids. Multi-grid iteration is a technique that communicates information globally in a very efficient way. It involves an averaging operator, to transfer information from fine grids to coarse, and an adjoint interpolation operator, to transfer information from coarse grids to fine.

In FY 93 we concentrated on developing the basic forms of the multi-grid and domain decomposition algorithms. These were programmed and tested for onedimensional advection-diffusion equations by Richard Hornung, as part of his thesis research under the direction of John Trangenstein. He also programmed a two-dimensional version of the algorithm for Laplace's equation.

In FY 94 Richard Hornung has extended the 2D algorithm to flow in porous media.

- He developed a discretization of the equations which is an extension of the standard lowest-order mixed finite element method. This discretization allows him to model contributions to the velocity components which arise from non-diagonal permeability tenscrs. He has developed a Gauss-Seidellike iterative algorithm for solving for pressure and velocity values using this discretization on a rectangular grid. During the Gauss-Seidel iteration the pressure equation is considered to involve only pressure as an unknown. 
- He developed appropriate interpolation and averaging operators for communications between levels of mesh refinement. During these parts of the algorithm, the pressure and velocity are treated separately. The averaging scheme for the velocity preserves total flow rates on the boundary of coarse cells, and the interpolation scheme for the pressure preserves Darcy's law across coarse-fine interfaces.

- An algorithm is being constructed for determining effective permeability tensors on the numerical grid. Effective permeabilities on a given collection of coarse grid blocks will be computed as averages of permeabilities on overlying fine grid blocks which enforce conservation of fluid flux across coars cell boundaries. Thus the multigrid averaging operator for the velocity has a very physical interpretation.

- He has written a Polymer equation of state $\mathrm{C}++$ class which places the two-dimensional version of the polymer flooding algorithm within the context of the adaptive mesh refinement code. The second-order Godunov method, which is used to advance the conservation law in time once we obtain a velocity field, is programmed and debugged. Detailed simulations of adaptively refined polymer floods will be conducted in the remainder of FY 94.

Figures 3 and 4 show the results of the multigrid algorithm on a mesh with irregular mesh refinement. Here the grid has been refined by a factor of two in four rectangular patches, two of which are contiguous. The pressure contours show very nice continuity across coarse/fine interfaces, to the extent that the contouring package allows the display. (Contour lines are only drawn using pressure values on the same level of mesh refinement.) The multigrid algorithm has been tested using several levels of mesh refinement, and refinement ratios greater than two. Extending the algorithm to dynamic relocation of the refinements is relatively easy (using the object-oriented code developed for the conservation laws), and is currently in progress.

\subsection{Analysis of Wave Behavior}

In FY 93 and 94, we examined two important problens: uniqueness of Riemann problem solutions for surfactant flooding, and Riemann problems for enhanced oil recovery problems involving hysteresis in relative permeabilities.

- Work on the analysis of Riemann problems for polymer flooding dates back to work by Pope in 1980 . In recent years, Johansen has analyzed the Riemann problem for simple surfactant models involving three components and 1-2 phases. He found cases where the solution is not uniquely determined by wave matching. This is potentially a problem for the work in this project, since we are using a surfactant model that, although more complicated, shares some of the same features as Johansen's model. During FY 93, Khaled Furati examined this problem as part of his dissertation 


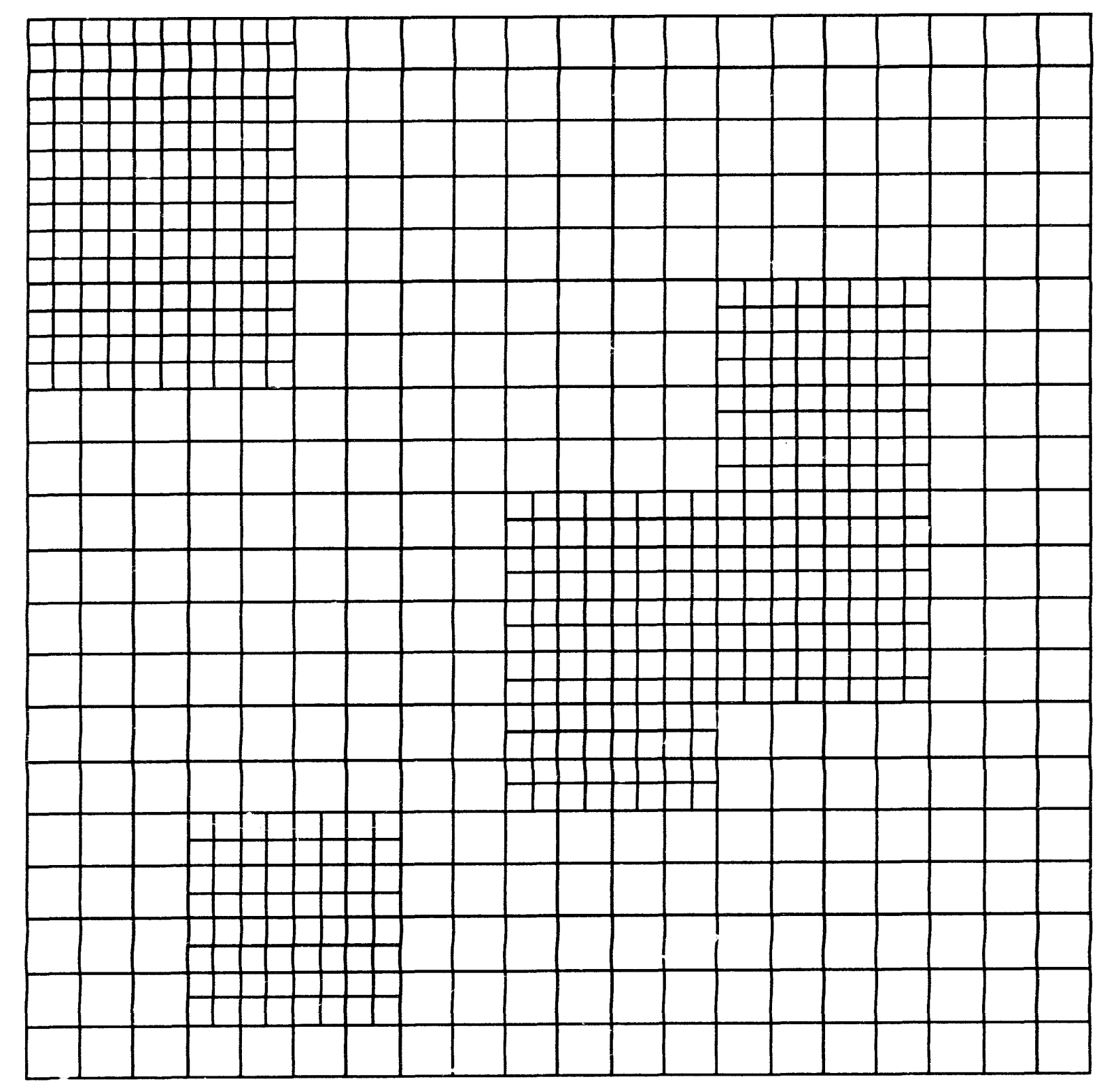




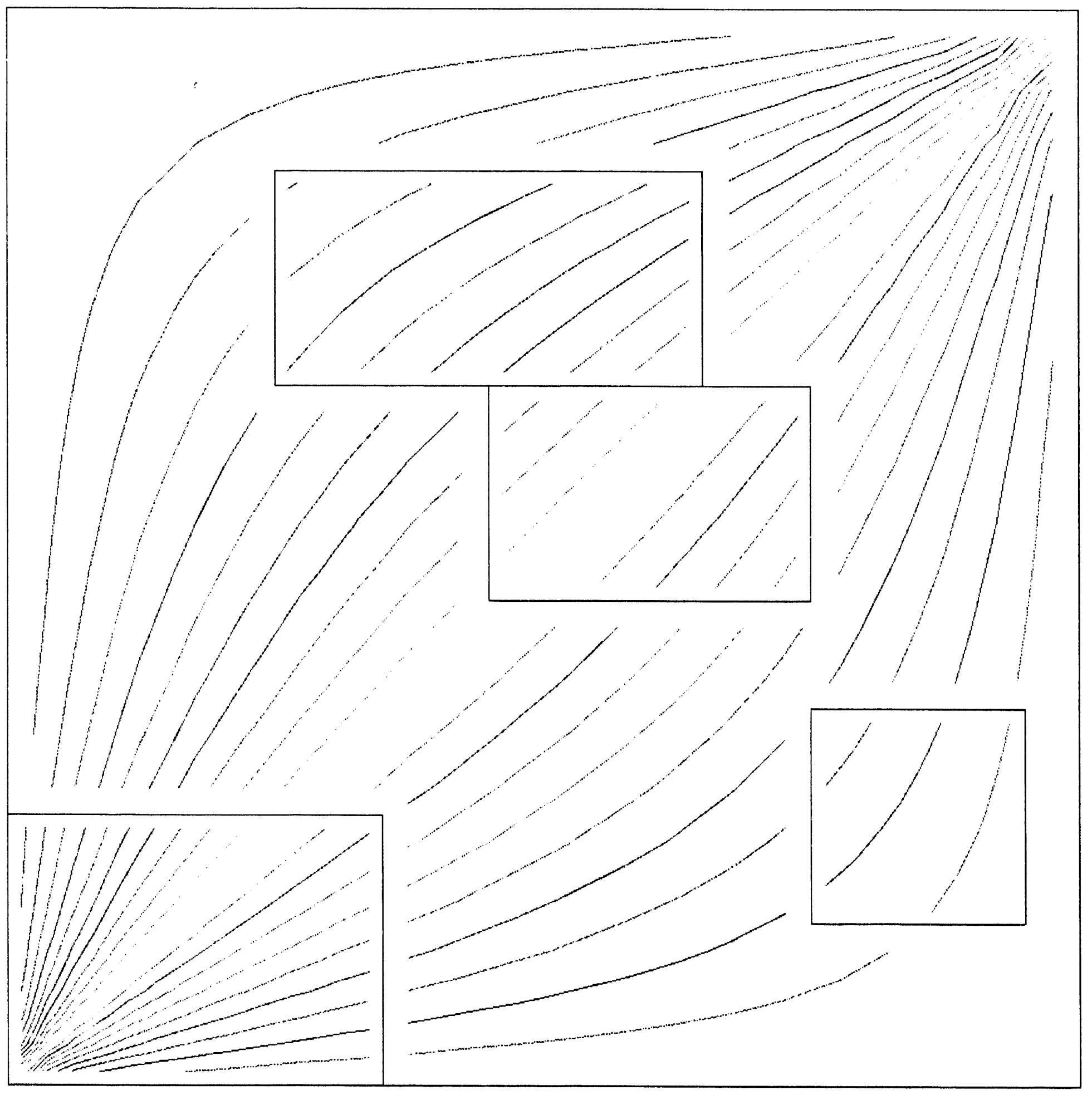


work at Duke. He was able to apply an argument by Smoller and Temple to show that only one of the solutions is obtained in the limit of vanishing viscosity. Thus this issue was completely and favorably resolved.

- Hysteresis in relative permeabilities is an important feature of multi-phase flow in porous media. Typically, reservoir simulation is conducted under the assumption that the saturations are either strictly increasing (e.g., water) or strictly decreasing. The simulators are then given the relative permeability curves for the appropriate imbibition or drainage behavior. However, many enhanced oil recovery processes involve the development of oil banks, in which the water saturation first decreases and then increases. This means that hysteresis could be important to the recovery predictions. The importance of hysteresis in contaminant spills is even greater. The spill process involves imbibition of the non-aqueous phase liquid (i.e., water drainage), and the cleanup process involves drainage of the non-aqueous phase liquid (water imbibition). The contaminant will exist at a range of saturations inside the aquifer, providing a variety of initial conditions for the cleanup process. In the second part of his thesis work (during FY 93 and 94), Khaled Furati has completed research into the effect of relative permeability hysteresis on the structure of fluid fronts in polymer flooding. The final results for the polymer model indicate that the inclusion of hysteresis effects can cause the oil slug to be not as high in saturation and wider in spatial extent than would be predicted without hysteresis.

\subsection{D Computations}

Under contract with Statoil during the spring of 1992, John Trangenstein developed a $3 \mathrm{D}$ polymer flooding simulator. This simulator used a second-order version of Godunov's method suggested by Jeff Salzman. Under that contract he extended the Salzman algorithm to multi-phase flow by incorporating a pressure equation and treating the velocity field as a spatially-varying quantity in the conservation laws. There were two wells: a rate-specified injector in one lower corner of the cubical reservoir, and a pressure-specified producer in the opposite corner. The injector well was designed to inject at constant water saturation and polymer concentration, and the pressure equation was solved only once, at initialization. The code was developed under a short contract, principally to prove that it could be done.

During the FY 93, John Trangenstein and Sameer Khan made some minor corrections to the $3 \mathrm{D}$ polymer code. They modified the injection strategy to inject a polymer slug followed by pure water without any taper. They also modified the code to solve the pressure equation for the velocity field at each timesiep. Although they could afford to run only on a relatively coarse mesh on our workstations $(17 \times 17 \times 17=4913$ cells for 7000 timesteps), the numerical results showed very nice resolution of the saturation fronts, and very little grid orientation. 
In the latter half of FY 94, the surfactant model will be extended to 3D simulations in a similar fashion. In this case, the injector and producer wells will be perforated in an entire column in order to reduce the number of timesteps required to flood the reservoir.

\subsection{Visualization}

Visualization is an important ingredient in scientific computation. Effective visualization tools can give the researcher important information about the state of the computation, and can be used effectively as debugging tools in certain situations. The combined use of color and shape is needed to give the mind maximum opportunity to obtain information quickly.

The choice and form of the visualization tools is important as well. For collaboration at a distance, portable and universal software is important, such as the XWindow system. For collaboration within a group, modular graphics software is useful to insulate stud nts from the details of the graphics internal operations. In this regard, the XWindow system is not very advanced.

- In FY 93 we wrote some 1D and 2D graphics tools, using the XWindow system and $\mathrm{C}++$, for special use with adaptive mesh refinement. For example, in 2D we draw the pictures by coloring coarse cells first and fine cells last as we examine the values on all patches on all levels of mesh refinement. Other modular aspects of the code allow the user to specify the name of variables desired for plotting on the command line, without having made any changes to the adaptive mesh refinement code at all. In this case, the plot requests are passed to the user-specified equation of state for processing. The mathematics students at Duke have found this graphics environment relatively easy to incorporate into their codes; as a result, even students working with other thesis advisors are using these tools. We also plan to make these graphics tools available to the Petroleum Engineering Department at the University of Texas. These tools were used to make the pictures in Figures 1-4.

- For 3D visualization in FY 93, we used the Iris Explorer system. This product shares many of the same features as the AVS system marketed by Stardent. Explorer is very simple to learn, and very effective for visualizing the 3D polymer flood described in the previous section.

- In FY 94, we have begun incorporating some of the Iris Inventor graphics into our simulation. We have been designated a beta test site for Inventor, and receive the latest versions of the software as it is developed. We began FY 94 by developing a prism tool to visualize the chemistry involved in surfactant flooding. This allows us to see the effect of salinity on the phase behavior of water, oil and surfactant. For example, the image hinted at in Figure 1 of the accompanying draft is displayed as the 3D image in the accompanying color image. The user is able to interact with the $3 \mathrm{D}$ image to adjust the viewpoint, while the simulation is underway. 


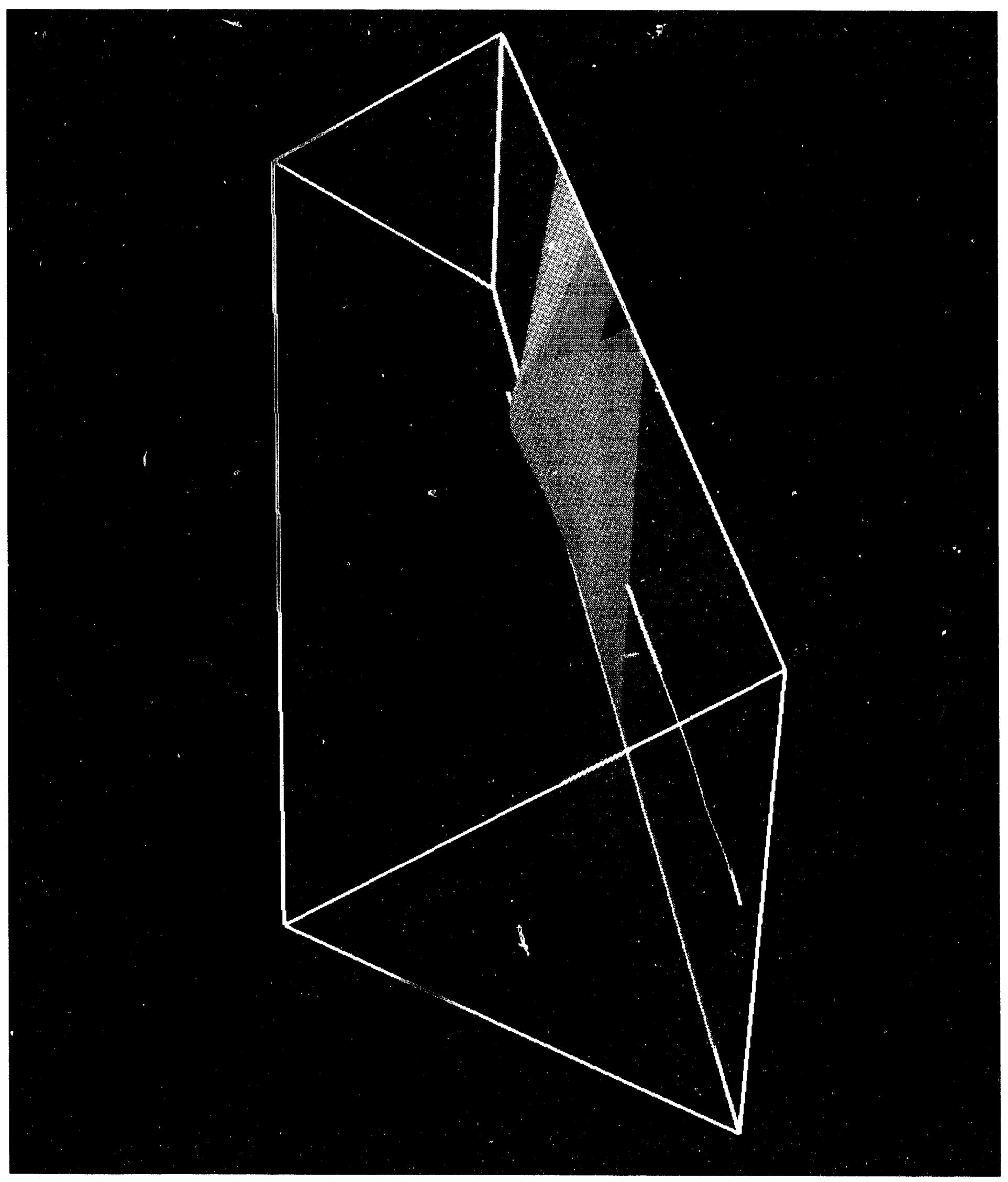


- Additional work will involve developing Iris Explorer modules to visualize the fluid properties This will give us quick graphic evidence of model discontinuities, and allow us to examine the qualitative aspects of the model for parameter determination.

This work is being conducted jointly by John Trangenstein, John Davies, Sameer Khan and Jon Simons.

\section{Future Plans}

During FY 95, we plan to continue our work on the surfactant model, adaptive mesh refinement and effective media properties, and graphics.

\subsection{Surfactant Model}

We plan to make extensive tests of the second-order Godunov method with adaptive mesh refinement in $1 \mathrm{D}$ and $2 \mathrm{D}$. In this effort, we will conduct numerical experiments to guarantee the reliability of the phase equilibrium calculations and to discover the structure of the fluid fronts. We plan to make extensive computational tests to examine mesh refinement and de-refinement strategies. We hope to compare our numerical methods to standard strategies in order to determine the relative efficiency of the second-order Godunov method to standard difference methods in the petroleum community. We would also like to determine the relative sizes of numerical dissipation and capillary pressure, in order to determine the conditions under which the latter is significant. Finally, we plan to continue the analysis of the characteristic structure of the flow equations. This work will be conducted principally by Sameer Khan and John Trangenstein.

\subsection{Elliptic Adaptive Mesh Refinement}

In FY 95, we will complete an efficient working version of the code and use it to perform extensive numerical tests. We will continue to analyze the discretization and iterative solution procedures in an attempt to support their validity with rigorous mathematical arguments. Then, we will verify any analytical results by performing numerical experiments on mathematically well-understood test problems.

We also plan to enhance our implementation of the pressure/velocity system solution process by exploring two additional areas. First, we will develop reliable error estimation procedures for the placement of refined grid patches. The hyperbolic solution process and the elliptic solution process can potentially employ very different error detection criteria. We hope to develop an approach which is suitable to both and which allows efficient dynamic placement of grid refinement during the coarse of a simulation. Secondly, we will test the appropriateness of different permeability averaging procedures in attempting to resolve the complexities of unstable fluid fronts on various grid refinement scales. 


\subsection{Renormalization and Effective Media Parameters}

In FY 95 we plan to study heterogeneous permezibility fields with adaptive mesh refinement in order to determine effective permeability tensors dynamically. The program development will be completed in the second half of FY 94, allowing for detailed numerical studies throughout FY 95. We will begin with simple fluic inoòels, such as single-phase flow with tracers, and extend the work to multi-phase flow models, such as the Buckley-Leverett model, polymer flooding and surfactant flooding.

\subsection{Graphics}

In FY 95 we will not have the support of several of the previous people. John Davies will be called upon to handle more of the department systems programming tasks, and Jon Simons will graduate in May 1994. We anticipate minor extensions of the capabilities they have developed, but no major new projects.

\section{Budget Justification}

For the third year of this project, funds are requestcd to support the postdoctoral fellow (Sameer Khan), one graduate student (Richard Hornung), and the principal investigator (John Trangenstein). We have proposed that the combination of these grants pay for $100 \%$ of the cost of the post-doctoral fellow and provide a modest stipend for the graduate student. The numbers on the budget sheet represent the fraction of these costs when shared between DOE ('95 budget $=\$ 60,000)$ and NSF ('95 budget $=\$ 50,000$ ).

Funds are also requested for hardware and software support for the computing machinery used in this project. Hardware support for our three Iris Indy's is $\$ 1000$ per year, and software support for the six Silicon Graphics machines is projected to be $\$ 180$ / year for each of our six machines. Two of the machines, the Indigo R4000's, were purchased with 3-year hardware maintenance included, so no hardware support is requested for these machines at this time. The other machine is a three-year old personal Iris, for which the hardware maintenance is prohibitively expensive and has been dropped.

Funds are also requested for minimal travel and supplies.

\section{Educational Human Resources}

An important but difficult aspect of this project is its interdisciplinary role and resulting impact on student training. This project involves a synthesis between mathematics and engineering, so collaborations between mathematics and engineering departments are crucial to its success. The principal investigator has assembled a team of graduate students in mathematics at Duke University to work on these problems, as well as a post doctoral fellow (Sameer Khan) 
who graduated from the Petroleum Engineering Department at the University of Texas.

Dr. Khan has made himself essential to this project. He adds physical insight that both the principal investigator and his mathematics graduate students are lacking. Both the graduate students and the principal investigator consult with him regularly for advice on the physical processes.

In FY 94 and 95 these grants have helped to support Richard Hornung work on his thesis, by providing money that he would otherwise have to earn through teaching. These grants also supported Dr. Khan for the academic year; in the summer, Dr. Khan is supported by a consulting contract with Statoil.

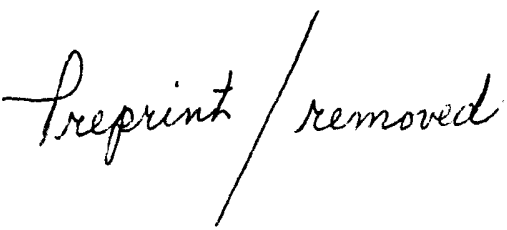



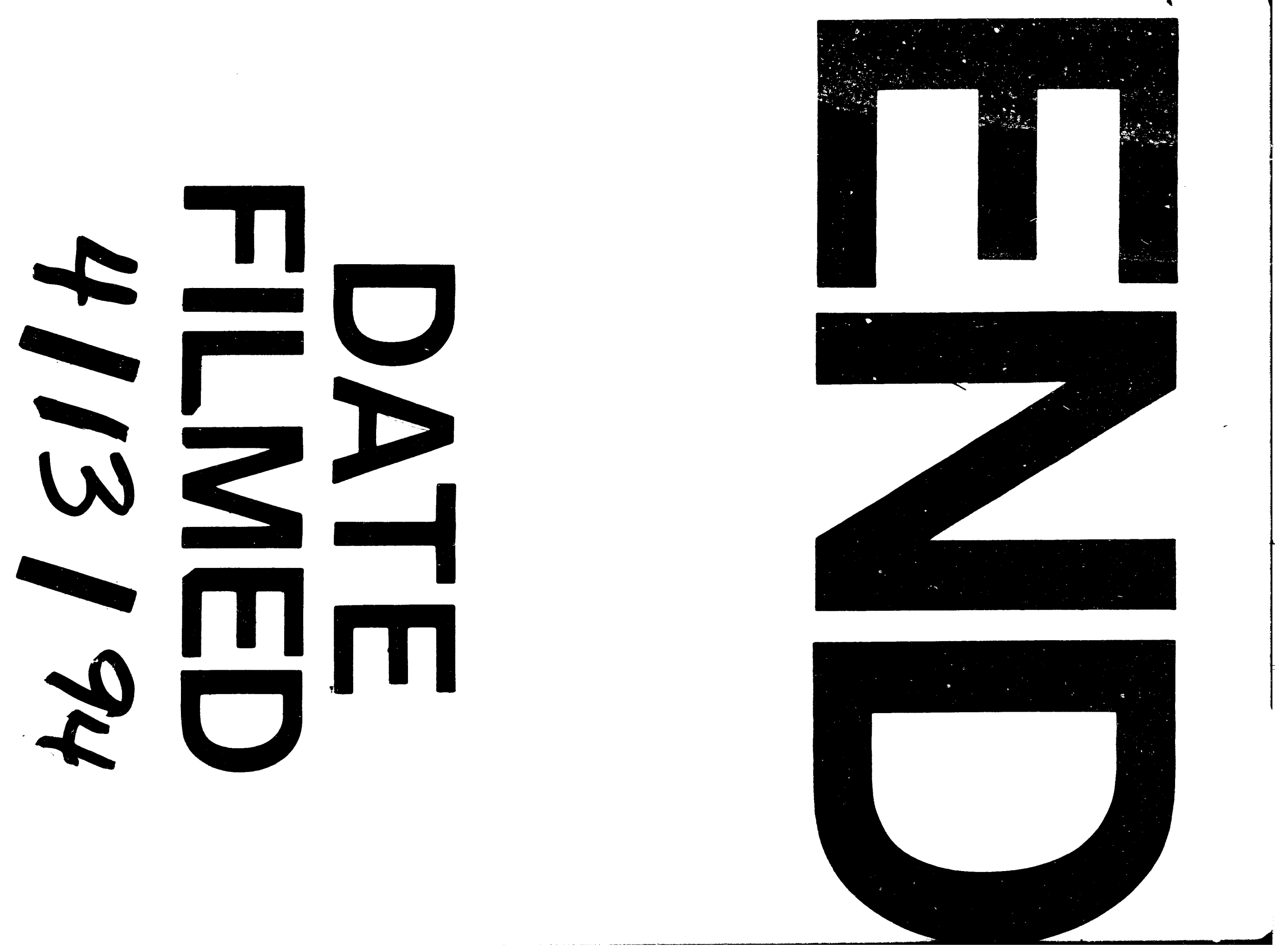


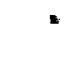

